\title{
Discussing on how to improve the subway rail flaw detection quality
}

\author{
Xu Haiwei ${ }^{1}$, Cao Jiangping ${ }^{1, a}$, Li Gang ${ }^{1}$, Wang Wei ${ }^{1}$ \\ ${ }^{1}$ Testing technology research institute machinery research institute of China, Cheng Du, \\ China ;ㅁ1878281644@qq.com
}

\section{Keywords: subway flaw detection}

Abstract: As the subway as public transportation in major cities in China play an increasingly important role, the subway operation safety more and more get people's attention.Due to the particularity of subway operation environment and status, makes the subway foundation, especially the quality of the steel rail.The purpose of this paper is to discuss how to better find that exist in the metro rail of hurt.

\section{Introduction}

Modern urban transportation system, the underground railway and urban light rail with its is not affected by climate conditions and characteristics such as safe, quick and efficient transportation, not only reduce the stress of the ground transportation, and brought convenient to urban residents life, so is get rapid development in our country.At present, in addition to Beijing, tianjin, Shanghai, guangzhou, Hong Kong's metro into normal operation, shenzhen, nanjing, Qingdao, changchun, Harbin, hangzhou, chengdu, chongqing and other cities of the subway today also is under construction and planning.However, the subway at the same time, bring convenience to people's lives, there is some problem that nots allow to ignore.

The subway as an important form of urban rail traffic, traffic density, small load, through a large amount, passenger comfort demands is high, the safety coefficient is high;Subway lines in the vast majority are in a tunnel, large buried depth, complicated;In addition, because the subway is mainly built on the ground below the rock or soil, it is not only influenced by in-situ stress and the effect of ground load, but also under the influence of underground environmental factors including seepage and damp.In this case, on the foundation of the subway operation, the quality of the rail has a bigger test.As the subway operation time, tunnel rail during the battle of reliability in the drab falling state, normal and safe operation that had an impact on the subway.Due to the particularity of subway construction and operation environment, if the subway operation after a period of time, to the rail for repair or replacement will be very difficult.With the development of social economy and people's material and cultural level enhancement, subways and light rails, as the representative of the emerging urban rail transit systems will get develop in modernization construction in the future.To the metro rail and its impact on the subject of tunnel structure reliability and durability research has become increasingly important and urgent.Develop advanced rail detection and protection methods, to reduce the subway construction investment, reduce operating costs, the subway will have a positive significance.

\section{Defects and causes of the subway rail}

Due to the use of special environment and condition, the subway rail metro rail defects including the legacy of the defects in manufacturing (white dots, bubble non-metallic inclusion, 
shrinkage cavity, segregation, etc.) and use the defects occurred in the process of two kinds, the latter is referred to as rail of hurt.Rail damage in use process refers to the rail fracture, crack and other effects and restrictions on rail use performance of the various states.Rail damage of formation is very complex, can be roughly divided into rail nuclear injury;Rail joint parts of hurt;Rail, rail bottom longitudinal horizontal and vertical crack crack.Currently existing in major metro rail damage including nuclear, scales, wound surface holes crack, metal shed and horizontal crack, transverse crack, etc.Because the subway operation has the characteristics of the traffic density, reciprocating wheel/rail friction, frequently lead to fatigue damage on the surface of the rail (such as scales, falling on the surface, etc.) more severe;Because the subway is currently in rapid expansion phase, line construction period, combined with the groundwater is rich in parts, part of the line segment waterproof project quality closes nevertheless, lead to the tunnel leakage, some places even drop directly to the rail surface, jaw cause rail, rail waist a corroded seriously; Short the distance between the subway station, the train start, braking is frequent, the impact of the wheel to rail welding joint, result in rail welding joint transfinite more etc. Wheel rail joint is the weak link line, effect on the rail joint of inertial force to around 30\% larger than the other parts of the largest, leading to joint parts of the rail head, rail waist rail bottom appear damage greatly increased.Also, the metro rail also exists in the process of manufacturing defects, such as rail longitudinal horizontal and vertical crack is due to poor rail manufacturing process, without removal of steel ingot with serious defects such as segregation, shrinkage cavity, inclusion, after the lake milk made from steel rail, defect flaky residue in rail head, rail waist, rail, and rail vertical parallel, in horizontal or vertical state;Vertical longitudinal crack also rail waist downwards into the bottom of the rail crack, rail rust pit bottom transverse cracks or scratches development form of rail, and in manufacturing steel rail, rail defects dairy or because of rail bottom plate between the sleeper is not close to stick, use rail in the local produce too much stress, cause rail bottom transverse crack or burst;And other adverse joint process, produce burnt, lack of penetration, air bubble, inclusion, and internal defects such as flare or resistant rail bottom transverse crack.

At present, the choice of stable performance of the rail flaw detection car or rail flaw detector for metro rail flaw detection, is the main way to detect the defects found in the rail.Detection vehicle inspection speed, strong adaptability, but poor flexibility, need artificial review after inspection.High detection sensitivity, good flexibility, but poor stability, affected by the operator human factors.Due to the complexity of subway lines crisscross, and the line is shorter, so commonly used detector on the rail flaw detection, the reliability of the test results depends mainly on the technical level of testing.Here are some of the ways to effectively improve the level of flaw detector to detect.

The importance of detection sensitivity for rail flaw detector, equivalent to the importance of accuracy for the gun.So analysis, choice of reasonable factors that affect the sensitivity of sensitivity, determine the best opportunity to adjust the sensitivity, flaw detector is the key to obtain accurate data.Depending on different conditions and rely on many years of experience accumulation, higher requirements to the operator level.Several examples below, with $70^{\circ}$ probe, to make its orbit the probe end echo a more clear and full, ensure displacement long enough, the front not clutter, in the process of pursuing rail flaw detection sensitivity, the greater the less said the better, better able to find small lesions;For $37^{\circ}$ probe, to ensure that the screw hole of the echo is clearly visible, displacement is long enough, in the absence of clutter, under the influence of the steel rail flaw detection sensitivity, the greater the said, the better, is conducive to timely found that unilateral fine holes crack and rail low transverse crack.In addition, with $37^{\circ}$ after probe, make sure of rail bottom echo and the screw hole is clearly visible and full, in the case of a waveform display and alarm, the 
lower rail flaw detection sensitivity, said, the better, is conducive to the rail longitudinal crack detection.

For close to the rail head lower slope face a small nuclear damage, we can take $70^{\circ}$ probe scanning.No Angle and Angle) can be used to scan and scan of the two methods, this article is only to do a brief analysis of the Angle scan way: $70^{\circ}$ probe in the various inspection training textbooks, guidance document has clear stipulation on the Angle, but its location on the surface of the on-orbit detailed rules, do not make everyone become generally to adjust it to the center of the rail head position, some inspection work for pursuing nuclear less injury of the wave high forceful clutter will its adjustment is by rail, the purpose of this way, a wave, a wave of obvious advantages, but the scan range is relatively narrow and easily missed rail is close to the center of hubei small nuclear damage.In order to effectively monitor the near the rail center of hubei small nuclear damage, generally within a to $70^{\circ}$ from the probe to adjust outside side a third rail head (wafer center distance rail head laterally about $25 \mathrm{~mm}$ ), make the ultrasonic wave acoustic distance increase.Half beam spread Angle, the rail head jaw after reflection, secondary wave scan area increase, hubei found rail site nuclear ability enhancement.Similarly, in block $4 \mathrm{~mm}$ man-made nuclear without residual, adjust to $70^{\circ}$ a probe to the rail side a third place in the head.As shown in figure 1 .
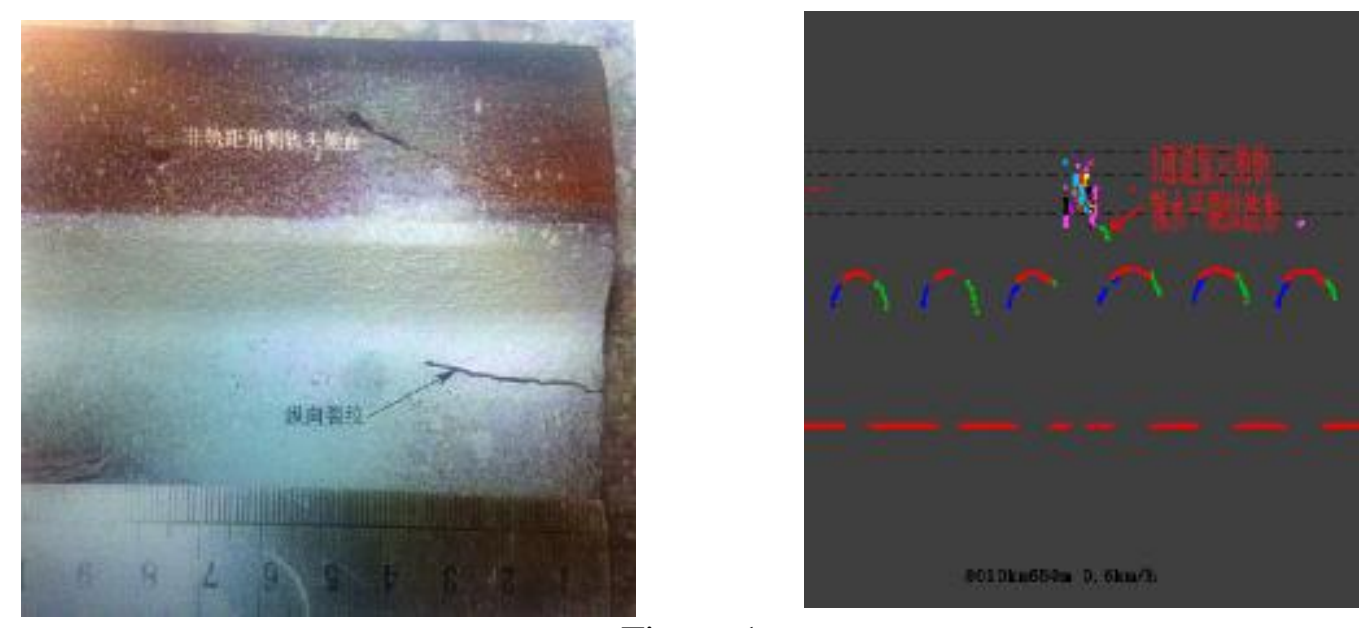

Figure 1

The bottom of the rail center transverse crack is one of the problem in rail flaw detection.It is hard to find, 2 it is difficult to determine.A solve method is in ZZTS rail eccentric block, $25 \mathrm{~mm}$ transverse crack $80 \%$ based on the wave height increases $3-6 \mathrm{db}$ as $37^{\circ}$ probe detection sensitivity; 2 it is to determine the base transverse crack, to probe the incident at measurement position, in the protective film on the gimbal ring sign out or probe, $37^{\circ}$ probe found rail base wave, probe the incident point to the defect of horizontal distance calculation, and use the ruler is marked on the rail.Look around $37^{\circ}$ probe whether coincidence detection position, such as the position is not on the sleeper, small mirror observation of rail bottom are available, and see whether there is a scratch, pit corrosion, grooves, ribbed, grease, etc. The defect waves (at the top of the rail side wave, on the other side without wave, namely before and after $37^{\circ}$ probe is only one common at the time of the wave) as these factors can be ruled out, there is the basic of the base can be thought of injuries. Such as the position of wave in the place such as sleeper is not observed, in addition to $37^{\circ}$ before and after using the position wave superposition method appraisal, also can use single $70^{\circ}$ probe from the upper rail.Detection must remove protective film, use the method with common flaw detector $\mathrm{k} 2.5$ probe, in the bottom of the rail center when the transverse crack in the screen have obvious displacement wave.As shown in figure 2. 

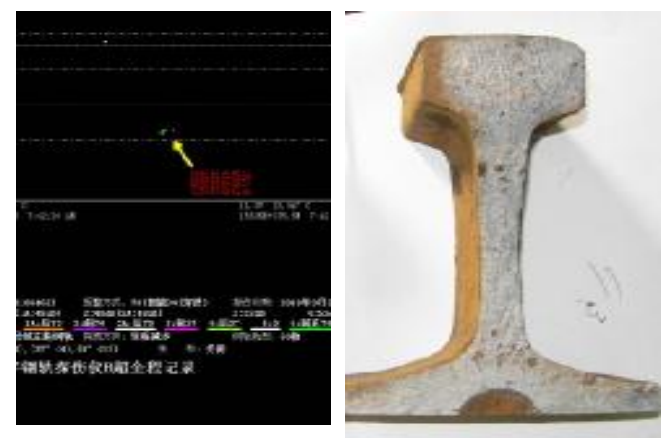

Figure 2

Side rail joint rail waist level crack monitoring is a difficulty, $37^{\circ}$ probe and $0^{\circ}$ probe are not easy to find $.0^{\circ} 1$ hole probe in orbit end to wave, $37^{\circ}$ probe lost or out of the wave clutter, but will try to adjust to the GTS - $600^{\circ}$ probe gain block $5 \mathrm{~mm}$ horizontal cracks and holes such as double $80 \%$ wave high $+6 \mathrm{db}$ on the detection sensitivity, increase water quantity, and the joint push it again.If $0^{\circ}$ probe in orbit waist wave, is to look on the rail side waist level or screw hole horizontal cracks, and is well versed in, notify the line work area overhaul when necessary.Rail parts of hubei level easily hide under the joint $70^{\circ}$ waveform figure, carefully identify. As shown in figure 3 level of rail surface crack detection waveform.
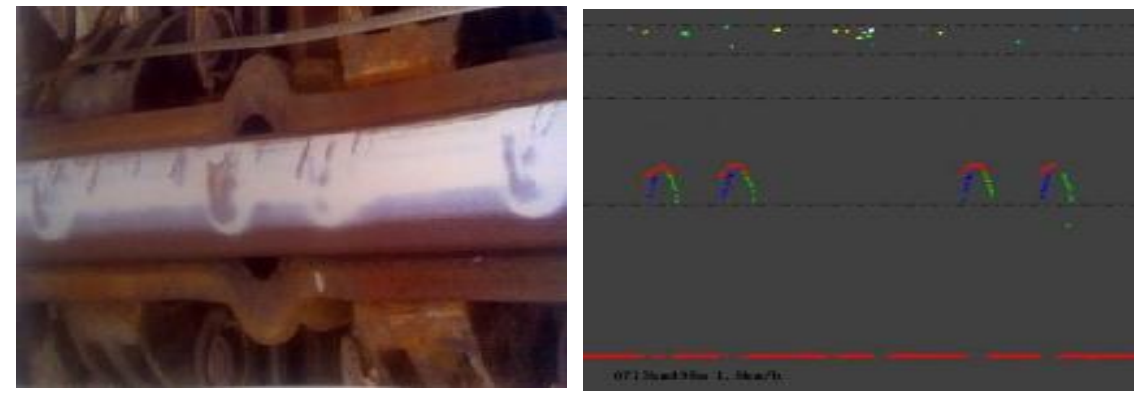

Figure 3

Welds rail waist level crack is rail steel condensation too fast in the process of welding, much in the winter construction site welding of thermit welds rail waist appear on one side of the weld reinforcement, and then gradually to rail waist internal development until the fracture, crack appeared in the early mainly by naked eye or magnetic particle inspection, after the crack to rail waist, $0^{\circ}$ probe in type A display on the screen of rail waist level echo of crack, at the same time, the base wave disappear and alarm;Show on-orbit jaw line party B, according to rail waist level crack pattern and Deborah.37 ${ }^{\circ}$ probe in the screw hole near the alarm door may show discontinuous, weak amplitude, a flash of mobile echo is gone;Type B shows present discontinuous points on-orbit jaw line.As shown in figure 4 thermit weld cracks and the Porto rail waist level.

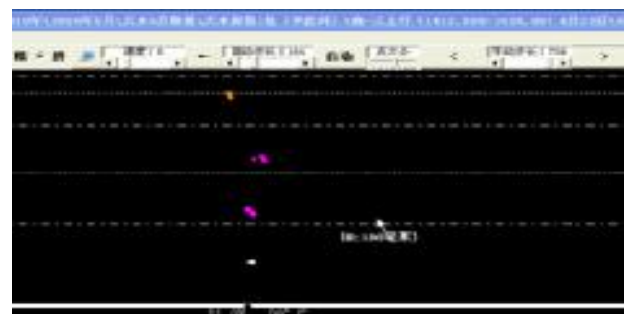




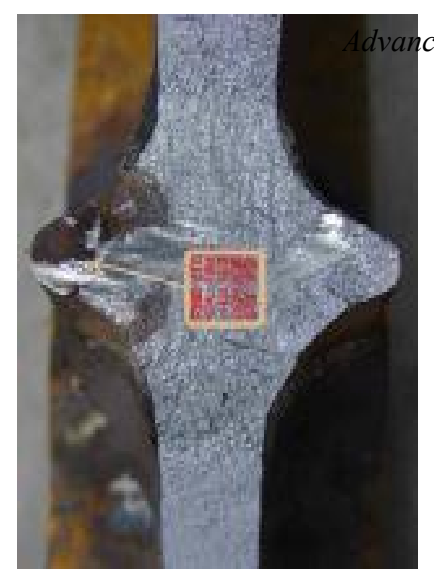

Figure 4

Rail end short feet refers to the rail joint rail end to the first hole center is not the size of the rail.In 60 rail, for example: the rail end to the first hole center is not the rail joint is rail end of the $76 \mathrm{~mm}$ short feet of rail joint, it's different from the normal rail joint B hypergraph is $37^{\circ}$ probe before rail stitches after half a screw Kong Bo ultrasound display graphical significantly longer, more than the normal screw Kong Bo, actually this screw hole wave shape is normal screw hole difform graph and the screw hole bobo charts which are formed by overlapping especially rail end saw when not in vertical cross section is more obvious. The launch of the sound waves in the tilt of the end face of $37^{\circ}$ after reflection, direction and wave type change, although there is a part of the sound energy after refraction returned to the probe, but increased the propagation distance, to bring back to pour dozen screw Kong Bo scale 5.0 or so, easy to be mistaken for screw hole horizontal crack to rail end, can through the visual difference between rail end method.

Often associated with short foot rail rail end rail end not neat, rail end not crack under vertical and rail end, need to be processed.Proofread the rail end short feet whether rail joint crack under the rail end, look at $0^{\circ}$ probe whether joint wave loss, if $0^{\circ}$ probe in orbit side wave, can improve on benchmark $37^{\circ}$ sensitivity gain $3 \sim 6 \mathrm{db}$, in guarantee instrument make rail end without clutter apex Angle e wave, reflected wave or rail is easy to observe them if there are other echo, according to rail end crack under general on-orbit end apex Angle reflection wave position slightly a little bit on location. $37^{\circ}$ probe rail end crack under B super graphics and rail end level B to exceed, graphics are on-orbit stitches this side have a crack at one end, and type B shows that first screw hole pour down the location of crack is different.

\section{improve the level of metro rail flaw detection}

Underground nuclear injury is a meticulous and complex work, its quality by the technical level of equipment, means and personnel determined comprehensively, to effectively improve the level of nuclear metro rail injury, must be Shared by different ways.

Strengthen quality management of detection

First, improve the management mechanism, specifically to do a good job in rail flaw detection, director of insight into the rail inspection work, and timely solve the problems arising from the rail flaw detection work.Second, the rail flaw detection equipment for irregular and regular inspection, so as to ensure the running of rail flaw detection instruments and equipment, ensure the quality of detection.Third, in strict accordance with the standards and procedures for testing and evaluation, the data should be strict censorship, strengthen the management level 3 data playback.Fourth, in the process of testing and evaluation, fully considering the environmental factors, reasonable arrangement of inspection plan.Fifth, the bad condition of rail location and track the number of abnormal, for two consecutive inspection cycle is found that the fatigue damage (such as nuclear injury, blue fish, screw hole crack, crack, etc.) level location check cycle shortened, avoid the 
occurrence of leak phenomenon.Sixth, do a good job, all sorts of rail damage record of minor track monitoring job should not be neglected, completes the data collection and integration, and analyze the rail flaw detection status, so as to find the general rule of the rail flaw detection.

Strengthen the equipment maintenance

Every month need on a regular basis for integrated maintenance, adjustment of the steel rail flaw detection instrument testing, to ensure that the instrument can be normal use.First of all, to probe frame frame, rail, etc., to those who have failed screws, springs for timely replacement and supplement, make rail probe Angle is correct, no looseness, adjust more flexible, ensure proper pressure, makes the function of each part as well.Secondly, regularly or irregularly to comprehensive sensitivity of testing instruments and probes how to performance, protective film change have failed in a timely manner.Finally, on the block of rail damage for debugging, fully grasp the dynamic condition of instrument, and make records.For reasonable adjustment testing work, require timely equipment checking and removing the malfunction, adjust the fixed detection range, appropriate baseline ratio and testing sensitivity correction.

Strengthen the management of rail flaw detection process

First, before the last inspection, need to debug carefully check all aspects of the performance of the instrument, such as voltage indicator, the combination of rail probe and protective film, by connecting with the probe pressure, water, etc., to ensure that the steel rail flaw detection instrument can work normally.Secondly, on the way, need according to the specific type and state of rail to adjust the location of the various channels alarm door, the position of the probe, and rail flaw detection sensitivity.Then, carry out, must strictly control inspection speed, catch each channel alarm sound, clear each channel waveform display, and carefully read all the probe's position on the rail, if appear abnormal display or alarm, then repeated detection, based on the waveform correction rail flaw detection sensitivity and location in a timely manner.Fourth, for the wrong tooth, insulating joint and split agent needs to be positive and negative direction, thus improve the gain, increasing water injection.Fifth, the rail head peeling impellers serious position, related testing instrument will failure, so you need to in strict accordance with the procedures, do a good job in all kinds of artificial cooperate with testing, avoid the occurrence of leak phenomenon.Sixth, according to the situation of rail damage of waveform display, many times to take a variety of probe and means, multi-level and check and verify, and not miss any information and location of a suspected, avoid the accident of the leak or wrongly, etc.In addition, in order to further improve the quality of rail flaw detection test, need to change the direction of the probe detection, alternating direction after the outside inside and outside the direction.In view of the detection curve grinding upper rail, need to adjust, thereby reducing the blind spot of track detection.

\section{Conclusions}

Rail damage is relatively serious problem in railway track traffic, directly affect the safety of the train operation and smooth.However in the process of long-term use, the rail will be broken, cracks and other damage impact performance, only clearly rail damage and its causes, to improve the quality of rail flaw detection work better.In general, in order to improve the quality and level of the steel rail flaw detection work, need to constantly sum up experience and lessons in the work, nip in the bud, in a timely manner to solve and deal with the problems and hidden dangers existing in the rail, so as to really improve the quality and level of the steel rail flaw detection. 


\section{References}

[1] Cheng Zhenzhong. An effective way to improve the quality of rail flaw detection - introduced in France VUR505 type car rail flaw detection [J]. Journal of railway construction, 1988 01:18 to 22. [2]Liu Tingting. Railway rail wound management research [D]. Beijing jiaotong university, 2009. [3]Jiang Haiyang. Railway rail damage of the design and implementation of management information system [D].Beijing jiaotong university, 2008.

[4] Zhao Xinchao. Study and design of ultrasonic flaw detector system hardware circuit and the echo The signal processing [D]. The northern industrial university, 2010.

[5]Gao Yunlai. Railway rail cracks online high-speed magnetic flux leakage inspection [D]. Nanjing navigation Empty space university, 2013.

[6] Shi Yongsheng quan-cai zhang, li jie, etc.Inspection car and flaw detector of rail head nuclear triage Comparative analysis on measured [J].Railway technology innovation, 2012 (1) : 99-101.

[7] Shi Yongsheng, Mr Li, Zhong Xufeng, etc.Rail tread oblique crack and rail head injury Type B figure analysis [J].Railway technology innovation, 2012 (1) : 92-95.

[8] The Chinese mechanical engineering society for nondestructive testing section.Ultrasonic testing [M].north Beijing: Beijing machinery industry press, 2000. 